\title{
ESTADÍSTICA ESTOCÁSTICA MULTIFRACTAL APLICADA AL ESTUDIO DEL HUASCARÁN EN LA CORDILLERA BLANCA $^{30}$
}

\author{
Gilberto Medina D.* Mauro Rodríguez C. ${ }^{1}$ y Abel Mejía M. ${ }^{2}$
}

\author{
*Facultad de Agronomía, Universidad Nacional Agraria de la Selva, Perú, gmeddiaz@gmail.com. \\ ${ }^{1}$ Facultad de Ciencias Forestales y del Ambiente, UNCP, mrcpe@yahoo.com \\ 2 Universidad Nacional Agraria La Molina, Perú, jabel@lamolina.edu.pe.
}

\begin{abstract}
RESUMEN
La pérdida de la masa glaciar de los nevados tropicales de nuestro país es tan notoria que hasta se le considera como un indicador del cambio climático que está sufriendo el planeta tierra. Según afirma el CONAM, para el año 2020, todos los glaciares por debajo de los 5500 metros desaparecerían. Una de las técnicas muy modernas a nivel científico es el uso de los multifractales, que permite medir probabilísticamente la magnitud de la superficie de objetos que crecen y decrecen en el tiempo. En este trabajo se realiza una aplicación de la técnica multifractal para estimar probabilísticamente el retroceso glaciar del nevado más alto de Perú (6768 msnm): el Huascarán, con un coeficiente de determinación mayor del $95 \%$. Asimismo, se mide su entropía y como aporte, hemos encontrado que los fenómenos El Niño y La Niña son percibidos en el espectro multifractal, indicando su clara incidencia de estos fenómenos físicos en el decrecimiento y en el mantenimiento de la superficie glaciar, respectivamente. Se utilizó el software Mass para el análisis multifractal e imágenes de satélite del lugar, con el índice de nieve NDSI y relación de bandas $3 / 5$. El objetivo del trabajo es analizar utilizando las técnicas tradicionales y multifractales, la desglaciación del nevado Huascarán en la Cordillera Blanca de Perú, así como medir la magnitud del área desglaciada desde 1987 hasta el 2009 y determinar la dimensión fractal empleando la relación de bandas $3 / 5$ y su entropía en el tiempo. Desde el año 1987 al 2008 el glaciar Huascarán ha disminuido de área en aproximadamente 8.6 \%. En la zona de Yungay, donde se ubica, la temperatura se ha incrementado aproximadamente en $0.08{ }^{\circ} \mathrm{C}$ y la precipitación disminuyó en $2.57 \mathrm{~mm}$ anuales. En conclusión, las modernas técnicas multifractales resultaron muy versátiles, prácticas y sensibles a fenómenos como el Niño y la Niña para medir el retroceso glaciar de nuestros nevados.
\end{abstract}

Palabras clave: Estadística multifractal, NDSI, relación de bandas 3/5, cambio climatico, sensores remotos, retroceso glaciar.

\section{STOCHASTIC MULTIFRACTAL STATISTICS APPLIED TO THE STUDY OF HUASCARAN IN THE CORDILLERA BLANCA}

\begin{abstract}
The glacier mass loss of tropical glaciers in our country is so noticeable that even is considered as an indicator of climate change on planet earth is suffering. CONAM asserts, by the year 2020, all glaciers below 5500 meters will disappear. A very modern techniques at the scientific level is the use of multifractal, which measures the magnitude probabilistically the surface of objects that wax and wane over time. In this paper, we apply the technique to estimate multifractal probabilistic glacier retreat of Peru's highest mountain $(6768 \mathrm{~m})$ : Huascaran, with a coefficient of determination greater than $95 \%$. Furthermore, entropy is measured as a contribution, we have found that EI Niño and La Niña are perceived in the multifractal spectrum, indicating a substantial effect of these physical phenomena in the decrease and maintenance of the glacier surface, respectively. Mass software was used for the multifractal analysis and satellite imagery of the place, with the rate of snow NDSI and band ratio $3 / 5$. The study aims to analyze using traditional techniques and multifractal, the deglaciation of Huascaran mountain in the Cordillera Blanca, Peru, as well as measuring the extent of deglaciation area from 1987 to 2009 and determine the fractal dimension using the band ratio $3 /$ 5 and entropy in time. From 1987 to 2008 , the Huascaran glacier area has decreased by approximately $8.6 \%$. In the Yungay area, where it is, the temperature has increased by approximately $0.08^{\circ} \mathrm{C}$ and precipitation decreased by $2.57 \mathrm{~mm}$ per year. In conclusion, multifractal modern techniques were very versatile, practical and sensitive to phenomena such as El Niño and La Niña to measure glacial retreat of our snow.
\end{abstract}

Key words: Statistical multifractal, NDSI, band ratio $3 / 5$, climate change, remote sensing, glacier retreat.

30 Trabajo de investigación fue recibido el 07/05/2010 retornado para su revisión el 15/06/2010 y aprobado para su publicación 16/1 1/2010 


\section{INTRODUCCIÓN}

Uno de los mayores problemas en la agenda contemporánea mundial es el cambio climático. Sin duda sus consecuencias para el planeta pueden ser catastróficas y es necesario tomar medidas para revertirlo, a la vez que para adaptarse a los escenarios que presenta. En esta nueva agenda el calentamiento global ocupa un lugar central [1].

En el contexto del cambio climático, los glaciares y especialmente los de nuestro país, por ser tropicales, son excelentes y muy sensibles indicadores de la evolución del clima; son ecosistemas vulnerables y constituyen las reservas sólidas de agua dulce, cuyos aportes, contribuyen a regular las descargas de las cuencas glaciares, que son utilizadas para fines múltiples, como el consumo poblacional, agricultura, hidroelectricidad $(80 \%$ de la energía en el Perú), actividad minera y otros; juegan por lo tanto un rol vital en el desarrollo socio - económico y aspecto ambiental - turístico de los países [2].

Según el Consejo Nacional del Ambiente (CONAM) [3], la superficie total de glaciares en el Perú se redujo en un $22 \%$ en los últimos 35 años. Como consecuencia de esta merma, hemos perdido alrededor del $12 \%$ en volumen de agua. Se estima que para los años 2015 ó 2020, todos los glaciales debajo de los 5 mil 500 metros van a desaparecer derretidos.

Una de las técnicas modernas para analizar los materiales como la nieve es el uso de los multifractales, . Por ejemplo se sabe que una hoja de helecho tiene una simetría qué responde a una ecuación que puede encontrarse en el campo de los números complejos. Los multifractales se emplean en el arte como en la pintura y en la música en la cual se generan imágenes y sonidos por iteración, así como en la ciencia donde mediante la técnica de la caja de conteo (box counting) permite analizar imágenes de satélite de tal manera que mediante una estadística muy sofisticada permite evaluar y caracterizar el grado de entropía y apreciar como fenómenos físicos como el Niño o la Niña actúan sobre las masas glaciares de nuestros nevados.

El objetivo de la investigación es la caracterización multifractal, cuantificar la desglaciación y determinar la dimensión fractal del nevado Huascarán.

Su importancia radica en que mediante el método de la caja de contar (box counting), podemos estimar estadísticamente su dimensión fractal, es decir hallar el número de
Besicovich, y observar en el espectro fractal las incidencias de los fenómenos del Niño y la Niña, según se trate la imagen satelital en estudio.

\section{Experimental}

\section{Área de Estudio}

El área de estudio abarca el nevado Huascarán ubicado en la Cordillera Blanca del Perú.

El nevado Huascarán abastece de agua a la ciudad de Yungay y su tendiente disminución afecta a la gestión integral de la Cuenca del río Santa.

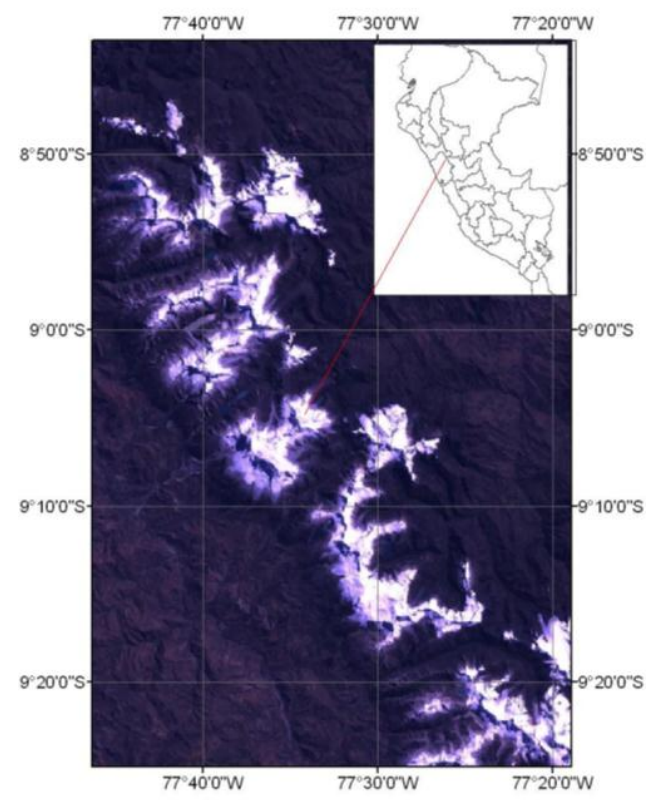

Fig.1 Cordillera Blanca en el centro del Perú. 


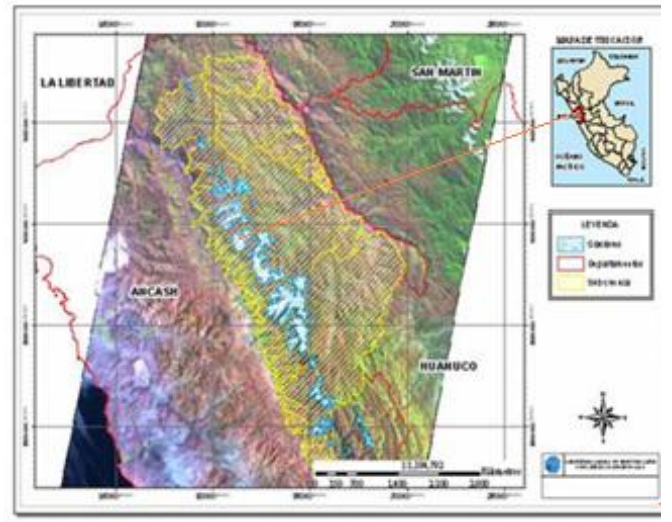

Fig. 2. Localización del nevado Huascarán en la Cordillera Blanca del Perú.

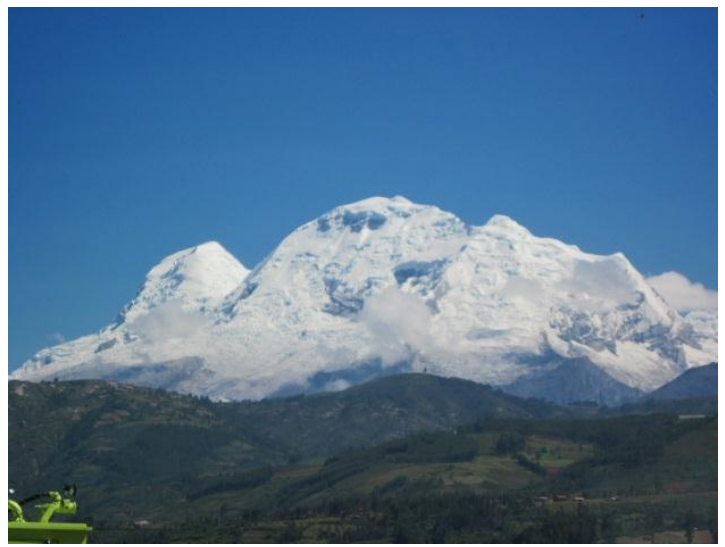

Fig. 3. Vista panorámica del nevado Huascarán.

\section{Acerca del Procedimiento 1}

Se consiguieron imágenes via internet del Instituto Nacional de Pesquisas Espaciais (Brasil).

Se georeferenciaron con la carta nacional a escala 1:100 000 .Se combinó en 3 bandas utilizando el software Erdas, Utilizando el Envi, se cortó la imagen a una escala redonda por ejemplo de 300x200 pixeles para el Huascarán.

Con el programa Erdas se separó las bandas y luego se halló el Indice Estandarizado de diferencia de nieve (NDSI) con la fórmula;

$$
N D S I=(b 2-b 5) /(b 2+b 5)
$$

Asimismo se halló la relación de bandas (ratio):

$$
\text { Band Ratio }=(b 3) /(b 5)
$$

Ambos métodos son excelentes para diferenciar la nieve de las sombras que producen las nubes, siendo el método del ratio imagen $3 / 5$ mucho mejor [4]..

En este trabajo lo usamos solo las bandas 2, 3 y 5 de varios años.

Fig 4. Bandas 2, 3 y 5 respectivamente del nevado Huascarán
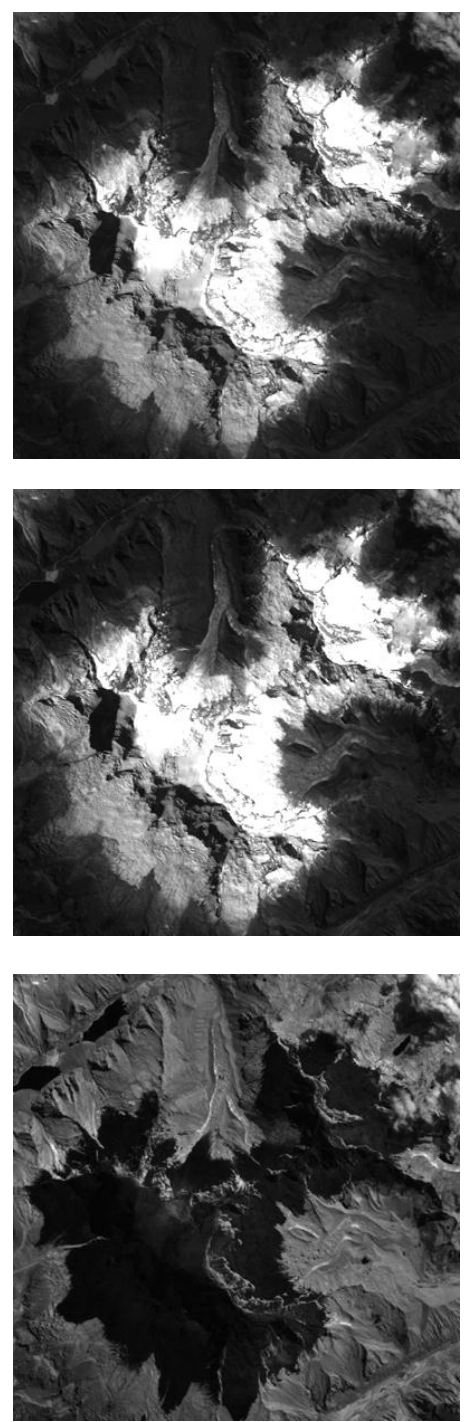

Las bandas 2, 3 y 5 se emplearon para hallar el NDSI y la relación de bandas o ratio $3 / 5$, y son frecuentemente usados para identificar la cobertura glaciar. 


\section{Acerca del procedimiento 2}

Comparando ambos métodos se escoge el ratio imagen por resultar mejor el perfil horizontal [5]. Con el programa Image j, binarizamos la imagen en ceros y unos y la llevamos al programa MASS y hallamos el espectro fractal..Exportamos la tabla al Excel y allí graficamos las gráficas a) alpha vs f(alpha) y b) Dq vs Tau, y luego determinamos la dimensión fractal.

$$
\begin{aligned}
& D_{0}=\lim _{\varepsilon \rightarrow 0} \frac{\log (N(\varepsilon))}{\log (\varepsilon)}, \\
& D_{1}=\lim _{\varepsilon \rightarrow 0} \frac{\sum_{i=1}^{N(\varepsilon)} \mu_{i}(\varepsilon) \log \left(\mu_{i}(\varepsilon)\right)}{\log (\varepsilon)}, \\
& \mathrm{D}_{2}=\lim _{\varepsilon \rightarrow 0} \frac{\log (C(\varepsilon))}{\log (\varepsilon)},
\end{aligned}
$$

\section{RESULTADOS}

\section{Clima en el entorno del Glaciar}

Para areas cercanas al glaciar Huascarán, durante el intervalo de tiempo de 1987 al 2008, apreciamos una tendencia creciente de la temperatura alrededor de $0.08^{\circ} \mathrm{C}$ y un decrecimiento de la precipitación de 0.9 $\mathrm{mm} / \mathrm{año}$ en el mismo periodo.
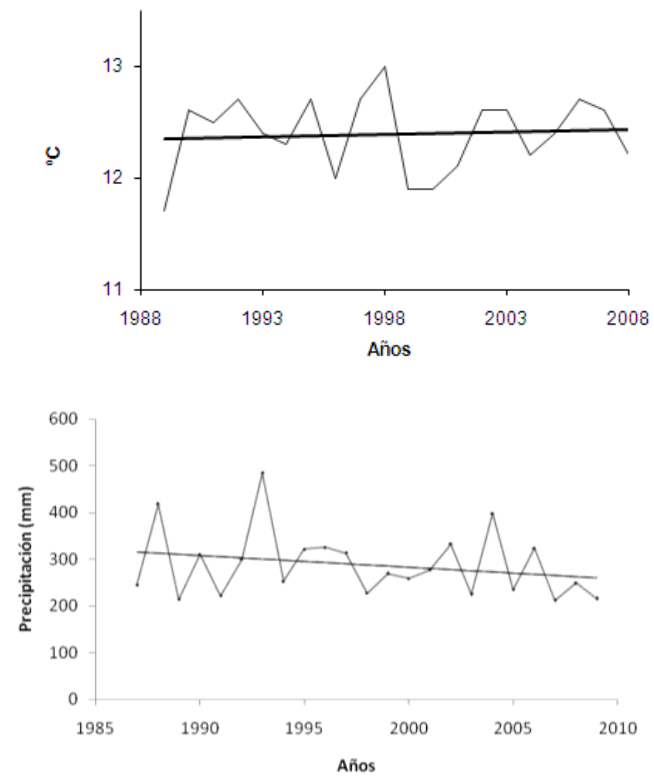

Fig. 5 Temperatura y precipitación en la estación Yungay, muy cerca al Huascarán.

\section{Cuantificación del área glaciar}

El área glaciar del nevado Huascarán (Tabla 1) ha disminuido en $8.6 \%$ en los últimos 20 años, esto es corroborado por [6].

TABLA 1: Pérdida de la superficie glaciar por métodos convencionales y multifractales

\begin{tabular}{ccccc}
\hline & ÁREA-SIG & & ÁREA-MULT \\
AÑO & $\mathbf{m}^{2}$ & $\%$ & $\mathbf{m}^{2}$ & $\%$ \\
1987 & 55344875.4 & 100 & 72982437.6 & 100 \\
1992 & 51707896.2 & 93.4 & 48563579.7 & 66.5 \\
1998 & 54138972.9 & 97.8 & 78942249.9 & 108.2 \\
2001 & 54560685.8 & 98.6 & 79345528.8 & 108.7 \\
2005 & 48169911.7 & 87.0 & 50292225.3 & 68.9 \\
2009 & 54322829.5 & 98.2 & 66738154.5 & 91.4 \\
\hline
\end{tabular}

Fig. 6 Pérdida de la superficie glaciar en el tiempo
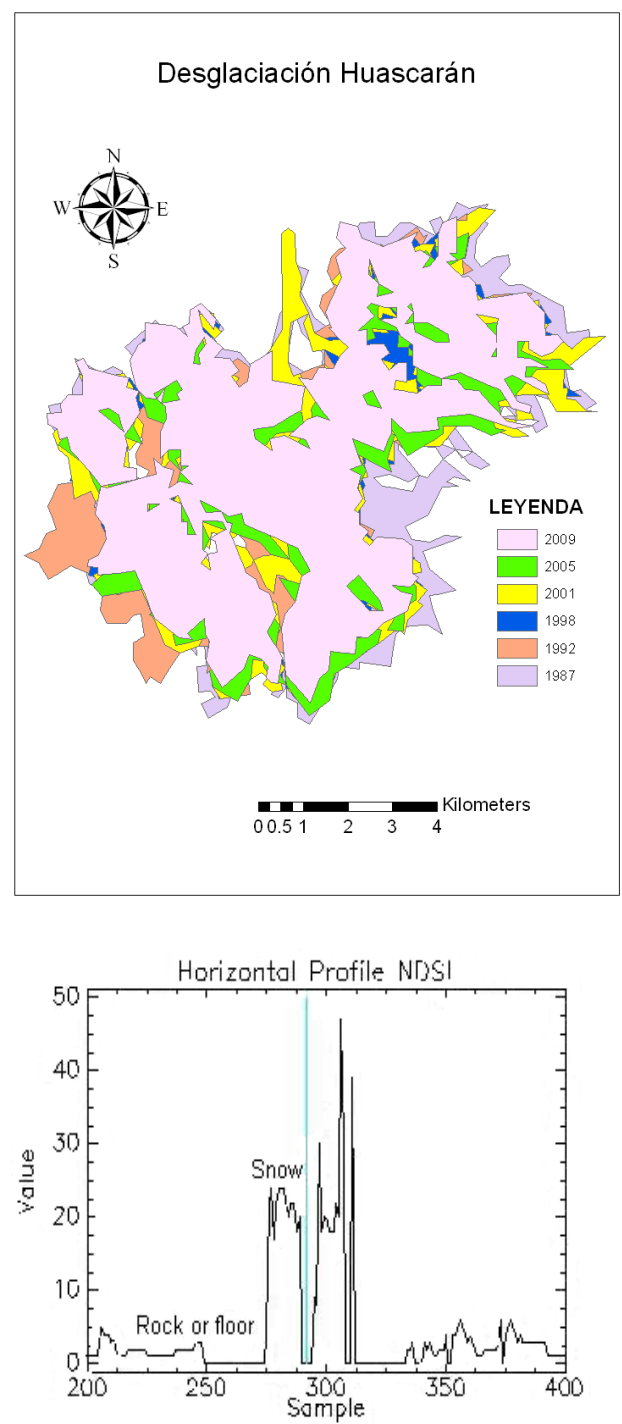


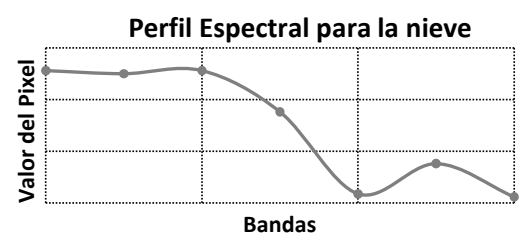

Fig 7. Perfiles horizontales del NDSI y del ratio $3 / 5$, respectivamente.

Como se aprecia en las fig. 7, 8 y 9 , la imagen del ratio $3 / 5$ resultó ser más uniforme para definir la cobertura glaciar [6] por lo que fue utilizado para hallar el espectro multifractal

Fig. 8 Relación de bandas 3/5 y NDSI

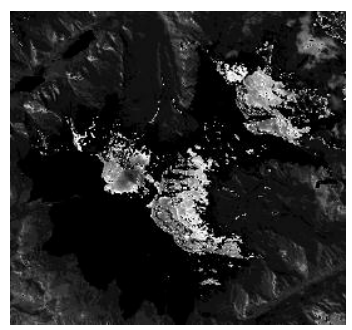

Fig. 9. Imagen RGB y perfil espectral
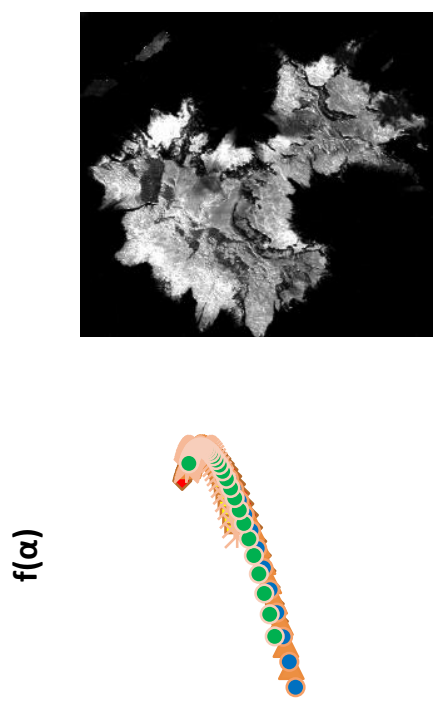

$\bullet 1987$

$\square 1992$

$\triangle 1998$

- 2001

$\times 2005$

$\alpha$

- 2009

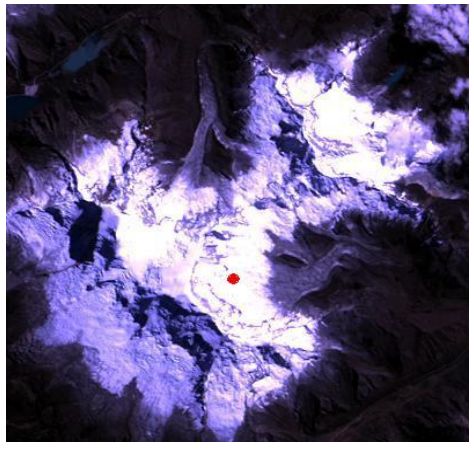

Fig 10. Espectros multifractales del Huascarán

El área glaciar se halló probabilísticamente con la Formula:

$P_{i}(\varepsilon) \sim \varepsilon^{\alpha_{i}}$

Dónde : $\boldsymbol{\varepsilon}$ es la escala y a es exponent de Lipschitz Holder.

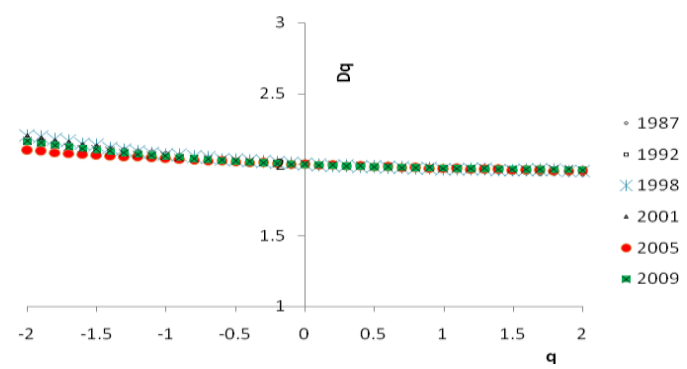

Fig 11. Función q vs Dq para el nevado Huascarán

En la Tabla 2 se muestra los parámetros básicos obtenidas a partir del espectro multifractal utilizando el software Mass.

Tabla 2: Dimensión capacidad $D_{0}$, dimensión entropía $\mathrm{D}_{0}$ y dimensión información $\mathrm{D}_{2}$

\begin{tabular}{llll}
\hline AÑO & $\mathbf{D}_{0}$ & $\mathbf{D}_{1}$ & $\mathbf{D}_{2}$ \\
\hline $\mathbf{1 9 8 7}$ & $\mathbf{2}$ & 1.95 & 1.93 \\
$\mathbf{1 9 9 2}$ & $\mathbf{2}$ & 1.98 & 1.96 \\
$\mathbf{1 9 9 8}$ & $\mathbf{2}$ & 1.97 & 1.96 \\
$\mathbf{2 0 0 1}$ & $\mathbf{2}$ & 1.98 & 1.97 \\
$\mathbf{2 0 0 5}$ & $\mathbf{2}$ & 1.97 & 1.95 \\
$\mathbf{2 0 0 9}$ & $\mathbf{2}$ & 1.97 & 1.96 \\
\hline
\end{tabular}




\section{DISCUSIÓN}

En el entorno del glaciar hubo un incremento de la temperatura y una disminución de la precipitación, lo cual concuerda con la bibliografía consultada [1].

El area glaciar del nevado Huascarán (Tabla 1) ha diminuido en $8.6 \%$ en los últimos 20 años [6].

La imagen del ratio $3 / 5$ observada en la figura 7 , presenta ondas más uniformes para definir la cobertura glaciar [6].

El escpectro multifractal de la fig. 10 nos indica que para los años 1987 y 1993 hay una pérdida mayor de masa glaciar, observándose una desviación hacia la derecha de forma asimétrica, debido a la presencia del fenómeno del niño, mientras que el año 2001 se presentó la niña, que permite un mantenimiento 0 recuperación de la masa glaciar, aquí su espectro es más simétrico. Para el año 2008 la masa glaciar ostensiblemente es menor que en los otros años, esto es corroborado por [8].

Los órdenes de momentos fueron usados para $q=-2$ a 2, fue encontrada tendencia lineal con un coeficiente de determinación $\left(R^{2}\right)$ mayor al $95 \%$.

La tabla 2, claramente nos muestra que $D_{2} \leq D_{1} \leq D_{0} \quad$ Aquí los valores de $D_{2}$ explican la correlación existente entre los valores medidos.

Para el año 1993 el rango de los exponentes de Holder (a), presentó su más alto crecimiento, esto es debido a la alta heterogeneidad que produce el evento El Niño (EEN) en el periodo 1991-1995. El año 2001 es uno con menos heterogeneidad (o mas homogeneidad) en el sistema glaciar, esto es debido a la ocurrencia del fenómeno La Niña (ELN), el cual crea una gran estabilidad en el clima y favorece el medio ambiente para la conservación de estados en el balance de glaciares [9].

\section{CONCLUSIONES}

- Las técnicas multifractales resultaron muy útiles para cuantificar probabilísticamente la superficie glaciar del Huascarán a partir de imágenes de satélite empleando la relación de bandas o ratio $3 / 5$.

- El espectro multifractal claramente refleja el impacto de los eventos El Niño y La Niña en la dinámica de formación del glaciar Huascarán.

- En el periodo 1997 al 2009 el area glaciar ha disminuido en aproximadamente $8.6 \%$, la temperatura en el entorno del Huascarán se ha incrementado en $0.08^{\circ} \mathrm{C}$ y la precipitación ha disminuido en $2.57 \mathrm{~mm}$.

\section{AGRADECIMIENTO}

Nuestro sincero agradecimiento al Centro Internacional de la Papa por habernos proporcionado el software Mass, para el análisis multifractal. A la Universidad Nacional Agraria La Molina, por el asesoramiento, y dirección

\section{REFERENCIAS BIBLIOGRÁFICAS}

[1] P. Chevallier, B. Pouyaud. y W. Suarez, W., Climate Change Impact on the water resources from the mountains in Peru. Paper presented at the OECD Global Forum on Sustainable Development: Development and Climate Change, OECD, Paris, 2004.

[2] J. Torres, A. Gómez, A. Adaptación al cambio climático, de los fríos y los calores en los Andes. Primera edición. Soluciones Prácticas-ITDG. Lima-Perú. 2008.

[3] CONAM. Escenarios climáticos futuros y disponibilidad del Recurso Hídrico en la cuenca del río Santa, Consejo Nacional del Ambiente. Lima - Perú 2005.

[4] A. Todd, Evaluation of remote sensing techniques for icearea classification applied to the tropical quelccaya ice cap, Peru" Cooperative Institute for Research in Environmental Sciences, University of Colorado at Boulder, Boulder, Colorado. 2004.

[5] C. Vargas, C. Villon, y J. Pasapera. Comparación de Técnicas para el Mapeo de Cobertura Glaciar con Imágenes LANDSAT y ASTER en la Cordillera Blanca, Ancash, Perú. Anais XIV Simposio Brasileiro de Sensoramiento Remoto, Natal, Brasil, INPE, 2009.

[6] M. Zapata. Cambio climático, deglaciación y sus implicancias en los recursos hídricos en el Perú. Resúmenes del I Congreso nacional del Agua, ponencia de la Unidad de Glaciología y recursos hídricos. 2009.

[7] F. Paul,A. KääB, y W. Haeberli,. Recent glacier changes in the Alps observed by satellite: Consequences for future monitoring strategies. Global and Planetary Change. 56 (2007), 111-122.

[8] M. Vuille, D. Hardy y R. Bradley, El impacto de los fenómenos El niño/la niña en la región del nevado Sajama en Bolivia" Dpto. Geociencias Univ. De Massachussets Amherst. 1998, 20 p.

[9] E. Ramirez, B. Francou, P. Ribstein, M. Descloîtres, R. Guerin, J. Mendoza, R. Gallaire, B. Pouyaud, y E. Jordan. Small glaciers disappearing in the Tropical Andes. A case study in Bolivia : Glacier Chacaltaya . Journal of Glaciology, 47 (2001) 157: 187-194 